\title{
Propuesta para el diseño de un código de ética empresarial basado en la ética kantiana
}

\author{
Proposal to design a business code of ethics based on kantian ethics \\ Proposition pour la conception d'un code d'éthique d'entreprise basé sur l'éthique kantienne
}

Florina G. Arredondo Trapero

Profesora Titular. Tecnológico de Monterrey, Campus

Monterrey. Monterrey, México.

Doctora en Economía y Dirección Empresarial,

Universidad de Deusto, Bilbao, España.

E-mail: farredon@itesm.mx

Lida E. Villa Castaño

Profesora Asociada, Departamento de Administración de Empresas, Pontificia Universidad Javeriana, Bogotá, Colombia.

Doctora en Filosofía Pontificia Universidad Javeriana,

Bogotá, Colombia.

E-mail: villa.null@javeriana.edu.co

Jorge De la Garza García

Profesor Asociado, Departamento de Mercadotecnia y Negocios Internacionales, Tecnológico de Monterrey, Monterrey, México. Maestro en Administración, Tecnológico de Monterrey,

Monterrey, México.

E-mail: jdelagar@itesm.mx

Artículo de investigación científica y tecnológica Según clasificación COLCIENCIAS

Recibido: $27 / 05 / 2014$

Revisado: 14/10/2014

Aprobado: 25/11/2014

\section{Resumen}

El objetivo de este artículo es determinar cómo los fundamentos de la ética kantiana pueden dar sustento a la construcción de un código de ética para una empresa concreta. La investigación es cualitativa, basada en entrevistas aplicadas a 138 colaboradores y analiza el proceso seguido en una empresa para diseñar su código de ética. En este proceso se respeta el principio de la autonomía del colaborador, tanto en la generación y apropiación de los criterios éticos. Bajo esta perspectiva, el código de ética logra establecer con claridad y precisión el deber ser de la organización siguiendo el criterio de la racionalidad y universalización de las normas, así como el respeto a la dignidad humana. Gracias a ello, el código de ética responde a los problemas que todo colaborador se enfrenta en la vida organizacional, así como asegurar su legitimidad como pilar de la ética empresarial.

\section{Abstract}

Palabras clave: código de ética empresarial, cultura ética empresarial, ética kantiana, ética empresarial.

This article sought to determine how the foundations of Kantian ethics can support the construction of a code of ethics for a specific company. This was a qualitative research based on interviews of 138 collaborators and which analyzed the process followed in a company to design their code of ethics. Within this process the principle of collaborator autonomy was respected, both in the generation and appropriation of ethical criteria. Under this perspective, the code of ethics manages to clearly and precisely establish the organization's ought be by following the rationality criteria and universalization of norms, as well as respect to human dignity. Due to this, the code of ethics responds to the problems every collaborator faces throughout organizational life, as well as ensures its legitimacy as a pillar of entrepreneurial ethics.

Keywords: entrepreneurial code of ethics, entrepreneurial ethics culture, Kantian ethics, entrepreneurial ethics.

\section{Résumée}

L'objectif de cet article est de déterminer comment les fondements de l'éthique kantienne peuvent supporter la construction d'un code d’éthique pour une entreprise en particulier. Il s’agit d'une recherche qualitative, basée sur des entretiens appliqués sur 138 collaborateurs, et l'analyse du processus suivi dans une entreprise pour concevoir son code d`éthique. On respecte sur ce processus le principe d’autonomie du collaborateur, si bien dans la génération comme dans l’appropriation des critères éthiques. Sous cette perspective, le code d’éthique est capable d’établir avec clarté et précision le devoir être de l'organisation en suivant le critère de la rationalité et l'universalisation des normes, ainsi que le respect vers la dignité humaine. Grâce à cela, le code d’éthique répond aux problèmes que tout collaborateur doit faire face dans la vie organisationnelle, en assurant en même temps sa légitimité comme pilier de l’éthique d’entreprise.

Mots clef: code d'éthique d'entreprise, culture éthique d'entreprise, éthique kantienne, éthique d'entreprise. 


\section{Introducción}

La ética empresarial además de formar parte de los criterios de decisión de los colaboradores que pertenecen a una organización, también es promovida explícitamente mediante la visión, la misión, los códigos de ética y los proyectos de formación del personal. Este tipo de documentos forman el patrimonio moral de una organización. En particular, en lo que respecta a los códigos de ética, estos son una ayuda para la resolución de conflictos, apoyo en la orientación de decisiones y clarifica responsabilidades, pues delimita los deberes y derechos de quienes laboran en las diversas áreas de la organización (Rodríguez y Díaz, 2004). El código de ética empresarial intenta integrar y converger la diversidad y heterogeneidad de las diversas formas de pensar de los colaboradores, generando así el concepto de un ethos corporativo (Cortina, Conill, Domingo y García, 1996). Por la relevancia que tienen los códigos de ética en la vida empresarial se plantea como pregunta de investigación en este artículo ¿cómo a partir de la propuesta de la ética kantiana se puede diseñar un código de ética para una empresa concreta? A partir de esta pregunta se propone como objetivo de la presente investigación, determinar cómo los fundamentos de la ética kantiana pueden dar sustento a la construcción de un código de ética para una empresa concreta.

Ahora bien, los códigos de ética se encuentran vinculados conceptualmente a lo que se denomina la deontología empresarial. Este ámbito se refiere en concreto a los deberes y obligaciones de quienes colaboran en una organización, así como de la organización entendida como un todo. Es decir, contienen aquello que es exigible al colaborador y a los directivos para el desempeño correcto de sus funciones tanto entre los colaboradores como en la forma de relacionarse con los stakeholders. Estos deberes normalmente son recogidos en un documento y aprobados por la alta dirección. También se establece que un código de ética empresarial es un documento formal que consiste en una serie de estándares morales empleado para guiar al colaborador de la empresa y a su conducta corporativa. Un código de ética empresarial no se trata de una serie de normativas que son impuestas y que resulten ser ajenas a la realidad de quienes forman parte de la organización. Todo lo contrario, un buen código de ética empresarial debe responder a la necesidad de un colectivo, al establecer los lineamientos básicos que favorezcan la interacción dentro y fuera de la organización (Rodríguez y Díaz, 2004).

\section{Marco teórico}

En las últimas dos décadas ha venido en crecimiento la construcción de códigos de ética en las empresas de todos los sectores. Se podría afirmar que tal auge es producto de los diversos escándalos empresariales los cuales han afectado directamente la imagen de la organización. De allí que, los comportamientos éticos que se dan en el seno de las organizaciones puede determinar hoy la confianza en términos de la selección de una organización a la hora de hacer inversiones. En este sentido, Ibarra (2002) indica que la relación entre la integridad personal y el desempeño laboral es un criterio muy importante que estan tomando en cuenta inversionistas para decidir en dónde invertir sus acciones así como clientes y proveedores potenciales e incluso organismos internacionales.

Los códigos de ética emergen entonces, como un instrumento mediante el cual se pueden delimitar las acciones de los individuos en relación con la toma de decisiones empresariales tanto al interior como al exterior de la misma. Al respecto, Schwartz (2001) realiza un estudio donde se puede analizar la relación entre los comportamientos de los individuos dentro de la organización en relación con el código de ética. Previo al estudio de los códigos de ética empresariales, es necesario realizar una aproximación conceptual a lo que es la ética y en específico a la relación con la empresa y los negocios.

\subsection{El concepto de la ética empresarial}

En la literatura actual encontramos diferentes perspectivas en términos éticos cuando ésta se refiere a las organizaciones. Por un lado, encontramos la ética organizacional que está referida al estudio de la conducta humana y cuyo objeto formal "es el estudio de la conducta humana en cuanto buena o mala para el individuo, el grupo o la sociedad" (Guillén, 2006, p. 8). En este sentido, la ética en las organizaciones se constituye como una ética aplicada que estudia qué tan humana es la organización lo cual se puede medir desde el desarrollo humano de quienes la integran.

Otro de los términos de la ética aplicada es la ética de los negocios o ética empresarial (business ethics) que adquiere relevancia cuando se indaga por la importancia de la ética en los negocios y como consecuencia de encontrar una cierta contradicción entre el objeto de la ética, búsqueda del bien común y los negocios, orientados a la maximización de utilidades, concebidos de cierta forma como amorales (Compte-Sponville, 2009). En este sentido, se comprende la ética en los negocios como una apuesta en el mundo empresarial en varios aspectos:

1. Comprensión de la estructura organizacional como un organismo moral (Cortina, 2003), esto indica que a partir de las acciones empresariales también se puede conseguir el bien común.

2. La necesidad de alinear los objetivos empresariales con el bienestar de todas las partes que que dan forma a la actividad empresarial. Esto implica un desplazamiento de la rendición de cuentas exclusi- 
vamente para los accionistas, a rendir cuentas en términos sociales, económicos y ambientales a todos los stakeholders que hacen parte de las decisiones que se tejen en la empresa.

3. Un reconocimiento de que la empresa impacta con sus acciones todo su entorno, por lo cual no es un ente aislado ni amoral. Por el contrario, al incluir la ética en la empresa como parte de su deber ser se empieza a perfilar una posibilidad de una sociedad más justa y equitativa.

Si bien la ética es una disciplina proveniente de la filosofía, lo que muestra la literatura más reciente es que la ética se aborda en la actualidad por diferentes disciplinas, como por ejemplo la contaduría, la administración de empresas y la economía, entre otras áreas, que dan forma al grupo de las éticas aplicadas. No obstante, su base teórica es filosófica como lo muestran autores como Velázquez (2010) y Weis (2011). En la presente investigación comprendemos a la ética en relación con la toma de decisiones y sus impactos sobre el entorno. De manera que en términos amplios, la ética tiene que ver con "las repercusiones que las propias acciones tienen sobre cada persona y sobre quienes la rodean, pueden ser consideradas explícitamente o ignoradas, pero lo que no se puede es impedir sus consecuencias" (Guillén, 2006 , p.17). En suma, cada que una persona o una empresa toma decisiones, se tienen repercusiones negativas o positivas sobre diferentes esferas, las cuales pueden ser económicas, sociales, ambientales y deben ser asumidas.

Ahora bien, la ética empresarial o la ética en los negocios empiezan a tomar relevancia cuando salen a la luz pública las acciones poco responsables gestadas en los centros de negocios y las empresas. Como por ejemplo, problemas de corrupción, maltrato a los derechos humanos, violación de los derechos laborales, afectación de la calidad de vida por daños al medio ambiente, etc. Esta serie de problemas pone en el centro del debate, tanto a nivel académico como empresarial, la necesidad de adoptar y acogerse a la ética como una forma de mejorar el entorno interno y externo de los trabajadores y de todos los stakeholders.

Si bien, históricamente se han propuesto diversas teorías éticas que suelen ser aplicadas a la ética empresarial, como lo es la ética de las virtudes de Aristóteles (2006), la ética del utilitarismo de Mill (2002), la teoría de la justicia de Rawls (2006), la ética del cuidado de Gilligan (1982), la ética de la responsabilidad de Jonas (1993), o la ética dialógica (Habermas, 2008) por mencionar algunas, es principalmente en la propuesta de la ética kantiana $(K a n t, 1921)$ donde se conjugan los pilares filosóficos que dan sustento a la construcción de los códigos de ética.

\subsection{La ética kantiana pilar del código de ética empresarial}

Kant (1921), el filósofo de Königsberg, construye su teoría ética apelando a la razón. En este sentido, los actos y las acciones del hombre deben surgir por la capacidad del razonamiento moral. Es decir, el razonamiento moral debe estar libre de prejuicios y de intereses egoístas y mezquinos. Las decisiones éticas buscan sobre todo el bienestar de la totalidad de los sujetos. De allí que, estas decisiones se funden en concordancia con el bien común.

Las acciones que realizan los sujetos se consideran éticas porque parten de una ley moral universal. Es decir, en razón a que la ética es la disciplina que estudia los estándares morales de uno mismo, o de la sociedad, estos estándares morales se establecen a través de un razonamiento lógico. Se es ético al actuar en conformidad con el bien y la integridad de los otros.

De modo que la teoría ética kantiana se fundamenta en un principio objetivo moral denominado imperativo categórico, el cual tiene dos formulaciones. La primera reza de la siguiente manera: "obra según la máxima que pueda hacerse a sí misma al propio tiempo ley universal" (1921, p. 91). La forma de interpretar la formulación anterior sería: no hagas al otro lo que no te gustaría que te hicieran a ti. La formulación kantiana entorno a la ética busca, por encima de las particularidades y de las subjetividades, un principio de universalidad en el que todos los seres humanos sean tratados de la misma forma, eliminando los intereses individuales que en últimas en ocasiones no son acordes con lo que requiere una sociedad justa y equitativa.

La segunda formulación que se considera que da el soporte para el despliegue de los códigos de ética es la siguiente: "cada uno de ellos debe tratarse a sí mismo y tratar a todos los demás, nunca como simple medio, sino siempre al mismo tiempo con fin en sí mismo" (Kant, 1921 , p. 86). De esta ley práctica se infieren elementos centrales para las éticas empresariales y los códigos de ética. Como por ejemplo, la dignidad humana. No usar la humanidad como un medio implica no cosificar ni instrumentalizar a las personas reduciéndolas a objetos de trabajo.

Por tanto, dignificar implica reconocer a los demás en cuanto persona. Es en este sentido que las decisiones éticas deben estar atravesadas por no afectar, ni vulnerar los derechos que todo hombre posee por el solo hecho de ser persona. Desde esta perspectiva teórica se comprende que las decisiones éticas que emergen de la interioridad y la individualidad buscan objetivos comunes a una comunidad o incluso en la sociedad. Se afirma que la ética kantiana es el soporte teórico de los principios éticos de los códigos de ética, en tanto si la dignidad humana es un principio rector para la realización de las acciones correctas. Con el hecho de cumplir este mínimo, de allí procederán los otros principios éticos fundamentales en 
las relaciones con los otros dentro de la esfera empresarial. Como por ejemplo, la sinceridad, la honestidad y la responsabilidad, el respecto por los derechos humanos, entre otros.

En síntesis la ética kantiana resalta nuestro estado de seres racionales, capaces de pensar y razonar. Tal como lo refiere Kant (1921, p.87) "la razón refiere, pues, toda máxima de la voluntad como universalmente legisladora a cualquier otra voluntad y también a cualquier acción para consigo misma, y esto no por virtud de ningún otro motivo práctico o en vista de algún provecho futuro, sino por la idea de la dignidad de un ser racional que no obedece a ninguna otra ley que aquella que él se da a sí mismo". Propone precisamente el fundamento de una ética centrada en la autonomía humana, en donde las leyes, normas, políticas o códigos no son impuestos irracionalmente sobre nosotros, de forma heterónoma sino que podemos razonarlas y apropiarnos de ellas como parte de un deber ser.

La razón es lo que nos separa de otros seres y nos brinda una conciencia que nos permite reflexionar sobre cómo debemos relacionarnos con los demás. La dignidad humana encuentra sustento en nuestra racionalidad. Se entiende que la dignidad humana es una cualidad unida al ser mismo de la persona, es decir, es la misma para todos. Es el valor que se descubre en el ser humano por el sólo hecho de serlo. Esta cualidad no se puede otorgar ni retirar, simplemente está presente en cada persona. La dignidad humana también impone ciertas actitudes y un comportamiento adecuado hacia las demás personas, fomentando el respeto entre unos y otros, y manteniéndonos en un nivel de igualdad en donde todos somos merecedores de los mismos derechos y libertades. Adicionalmente, tenemos que la dignidad humana nos otorga el derecho de ser tratados como personas, no como objetos, porque de acuerdo con Kant (1921 p. 88), "en el reino de los fines todo tiene o un precio o una dignidad. Aquello que tiene precio puede ser sustituido por algo equivalente; en cambio, lo que se halla por encima de todo precio y, por tanto, no admite nada equivalente, eso tiene una dignidad". Para Kant aquello que satisface necesidades tiene un precio comercial, o bien puede tratarse de un precio afectivo. Si bien las cosas sirven para resolver necesidades, no así las personas, que tienen la condición de ser fines en sí mismas, la persona no tiene meramente valor relativo 0 precio, sino un valor interno, esto es, dignidad.

El respeto a la dignidad humana debe ser el fundamento de toda relación y debe estar presente en nuestras interacciones con otras personas; así como las reglas que nos rigen, que deben ser universalizables, considerando que lo que es válido para uno, debe ser válido para otros. Es desde la teoría ética kantiana de donde se configuran los principios éticos rectores necesarios para construir relaciones que fortalecen los vínculos humanos en un entorno como el empresarial y que quedan reflejados en los códigos de ética organizacionales.

\subsection{Los códigos de ética}

Los códigos de ética funcionan como un marco de referencia para el comportamiento ético y suponen la elaboración, el consentimiento y la aceptación de las normas que el código contiene por parte de los colaboradores (Schwartz, 2001; Schwartz, 2004). Sin embargo, este deber ser al que deberían estar comprometidos los colaboradores, en ocasiones se encuentra muy distante de la realidad, basta mencionar el caso Enron (Hamilton y Francis 2003). Por una parte los directivos cometieron serias fallas éticas y por otra parte, a todo empleado se le obligaba a seguir un código de ética que debía ser firmado el primer día de trabajo (Helin y Sandström, 2007).

En relación con lo anterior, hay dos razones que explican por qué resulta tan difícil evitar las malas prácticas. La primera "hecha la ley, hecha la trampa" (Melé, 2014, p. 8). Es decir, sin voluntad ética, el colaborador busca los recovecos para burlar las normas. Y la segunda es la falta de sensibilidad y voluntad ética, porque "la moralidad no se queda en la letra de la ley sino en la justicia que la justifica” (Melé, 2014, p. 9). Por ello en la elaboración de un código de ética deben quedar claras las razones que justifican las normas, ya que en ello reside propiamente su valor.

Si bien un código de ética no es garantía de un comportamiento ético, en la actualidad son más las empresas que optan por tener esta herramienta como elemento rector de la acción correcta en las relaciones dentro y fuera de la organización. Sin embargo, los códigos de ética varían de acuerdo con el tipo de organización y el tipo de responsabilidad. La Tabla 1 muestra las cinco generaciones de códigos de ética (Mendes y Clark, 1996).

De acuerdo con la Tabla 1, podemos indicar que los códigos de ética varían en relación con la responsabilidad y el impacto de la empresa en la sociedad (Lozano, 2004). En este sentido, pueden existir códigos de ética encaminados a dirigir la buena acción de los empleados sin que exista una preocupación por el entorno o incluso sin considerar que el fortalecimiento con los grupos de interés, por medio de una relación ética, genera confianza en todas las partes involucradas. Este tipo de códigos de primera generación son simples en su constitución pues solo desvelan los principios rectores de la acción procurando el beneficio de los accionistas. De manera que, las acciones correctas éticamente no se proyectan como una necesidad de impactar positivamente en el entorno de la organización.

No obstante, a partir de la Tabla 1, también podemos afirmar que la complejidad de los códigos éticos está en relación directa con los objetivos estratégicos del negocio y de las responsabilidades empresariales. En este sentido, a medida que la operación empresarial se complejiza, el código ético será más exigente, en tanto éste involucra aspectos internos y externos de la actividad empresarial. 


\begin{tabular}{|c|c|}
\hline Generación & Características de los códigos de ética \\
\hline 1a generación & $\begin{array}{l}\text { Centrados en los conflictos de interés este tipo de códigos tiene como objetivo proteger a la } \\
\text { empresa de las decisiones poco acertadas tomadas por los empleados. El código de ética más que } \\
\text { seguir principios éticos busca la supervivencia en el mercado y el beneficio de los accionistas. }\end{array}$ \\
\hline 2a generación & $\begin{array}{l}\text { Estos códigos están centrados en mostrar cómo desde los principios éticos (transparencia, } \\
\text { honestidad, responsabilidad, etc.) se fundamentan las relaciones entre las personas que trabajan } \\
\text { dentro de la organización. El código de conducta se constituye en una guía para la acción correcta al } \\
\text { interior de la organización. }\end{array}$ \\
\hline 3a generación & $\begin{array}{l}\text { Amplio reconocimiento de los grupos de interés, lo que implica que las empresas que siguen este } \\
\text { tipo de códigos saben que sus acciones tienen efectos al interior y al exterior de su entorno. El } \\
\text { concepto de gerencia es amplio ya que se involucran temas necesarios en el ámbito empresarial, } \\
\text { como por ejemplo, los derechos humanos. }\end{array}$ \\
\hline 4a generación & $\begin{array}{l}\text { En este tipo de códigos la empresa tiene en cuenta el medio ambiente, los stakeholders directos y la } \\
\text { comunidad. Son las empresas que tienen grandes impactos sobre el medio ambiente, como por } \\
\text { ejemplo las mineras y las petroleras, las que utilizan estos códigos de ética para regular las } \\
\text { acciones, en procura de no impactar dramáticamente este ámbito, al igual que a la comunidad } \\
\text { próxima a excavaciones. }\end{array}$ \\
\hline 5a generación & $\begin{array}{l}\text { Utilizados por empresas multinacionales y transnacionales. Son los más exigentes en tanto además } \\
\text { de incluir los aspectos medioambientales, las relaciones con los stakeholders y la comunidad } \\
\text { involucran la cadena de valor de la empresa y el modo cómo se debe invertir en cada país. De } \\
\text { acuerdo con Porter y Kramer (2006) este tipo de códigos incorporan de manera estratégica los } \\
\text { ámbitos relevantes de la responsabilidad social empresarial. Estos son: los derechos humanos, los } \\
\text { derechos laborales, el medio ambiente, la publicidad y el mercado de impacto en el desarrollo y la } \\
\text { transparencia. En este sentido, Longinos, Arcas, Martínez, Olmedo (2012) mencionan que hay cierto } \\
\text { tipo de códigos de conducta que hacen referencia a la transparencia, entendida como la claridad } \\
\text { con la que una organización expone los resultados de su gestión a los diversos grupos de interés. }\end{array}$ \\
\hline
\end{tabular}

Para Hartman, Desjardins y Espinoza (2011) una cultura basada en el cumplimiento valora la obediencia a las reglas como la obligación primaria de la ética por lo que obliga y monitorea el cumplimiento de la ley y de los códigos internos. Lo contrario a una cultura de cumplimiento, es una cultura organizacional basada en valores, en donde se elaboran códigos de ética aspiracionales, flexibles y visionarios. Por otra parte, Gopinath (2008) argumenta acerca de las dificultades en relación con la estandarización de los códigos de ética a diversos contextos culturales. Las prácticas correctas en el entorno empresarial varían de un contexto cultural a otro, por lo que es difícil contar con un código de ética empresarial único cuando se trata de empresas globales.

Finalmente, más allá de la complejidad de las generaciones de los códigos de ética, se debe comprender que el código de ética, entendido como el ADN empresarial, no solo genera cultura organizacional, sino que contribuye a la construcción de una mejor comunidad (Comte-Sponville, 2009).

\subsection{Códigos de ética aplicados a la organización}

Si bien los códigos de ética no aseguran una racionalidad convencida de todos aquellos que laboran en la organización, sí logran proporcionar un sentido colectivo de aquello que resulta ser el ethos organizacional, como lo aclara Ro- dríguez y Díaz (2004, p. 43) "un código de ética no logra que los empleados sean intrínsecamente morales, pero sí activa el sistema de valores de una organización, proporcionando un sentido colectivo de lo bueno y de lo malo".

Además de los códigos de ética, las empresas manejan una amplia variedad de mecanismos para enfrentar los retos éticos. Algunos de ellos son la ética corporativa y mecanismos de gobernanza corporativa, la protección a quienes denuncian internamente actos de corrupción y así como criterios de promoción a quienes se conducen con integridad (Rodríguez y Díaz, 2004).

Los códigos de ética forman parte de las estrategias formales orientadas a fomentar la ética en la organización y su proceso debe seguir un orden lógico (Argandoña, 2003). Como parte del proceso de la definición e implementación de un código de ética organizacional, una vez realizado el diagnóstico, definido el qué y alineada la acción, es importante que al revisar el proceso, se hagan las adecuaciones pertinentes. Este último paso permitirá que la cultura ética en la organización vaya hacia mejor.

En relación con lo anterior, Arredondo, De la Garza y Vázquez (2014) estudiaron la influencia de los códigos de ética en cuanto a la normatividad de una organización, su aplicabilidad y difusión como parte de la estrategia de una organización que busca la transparencia. En sus hallazgos establecen que las empresas privadas suelen revisar los códigos de ética y adecuar posibles cambios en el documen- 
to, a diferencia de las empresas públicas, que saben de la existencia de un código de ética en su organización, pero ignoran los procesos de revisión y adecuación que se siguen en algunos casos.

Definir el "qué se quiere ser" como organización en términos de ética, requiere de un trabajo en equipo formado por la alta dirección y el consejo consultivo (Argandoña, 2003). La estrategia de preguntar a todos los colaboradores qué principios éticos debería adoptar la compañía, es seguir un proceso exhaustivo y no eficiente, porque no todos sienten a la empresa como propia, ni todas las contribuciones al contenido del código tienen la perspectiva completa de la organización. Ciertamente en toda organización hay colaboradores que no están motivados en plantear estrategias para promover una cultura ética en la organización. Sin embargo, es importante que en el proceso de definición, los diferentes niveles de la organización estén representados.

Rodríguez y Díaz (2004) refieren que los códigos de ética deben ser producto de una construcción colectiva, por lo que no deben limitarse a ser una declaración de valores, sino que deben generar un compromiso real y acciones tangibles en aquellos que integran la organización. Los estándares éticos que se imponen desde la élite directiva pueden llevar más tiempo para integrarse al quehacer de la organización. No siempre el colaborador se compromete con la ética de la organización, porque desde un inicio no se ve representado en ella.

Por esta razón para Pou Munt, Fabbri, Chamón, Villavicencio y Schmidt (2014, p. 4) el código de ética debe ser una expresión de lo que los integrantes de una empresa desean vivir, por ello es importante un proceso que "ayude a las personas a descubrir, aclarar y perfeccionar su propia cultura institucional". De otra forma, tal como lo advierte Vieira (2012), un código de ética que se elabora para aplicar sanciones puede tener un efecto contraproducente en la organización, puede provocar rechazo y además correr el riego de representar solamente un sistema de control en la empresa (Pou Munt et al. 2014, p. 5). Por ello, todo código de ética es importante que emerja de la propia conciencia de las personas, que refleje lo que desean ser y la forma de trabajar como parte de su cultura organizacional.

\section{Metodología}

El estudio se desarrolló a partir del análisis de la experiencia en la generación de un código de ética en una empresa de la industria de alimentos en la ciudad de Monterrey, Nuevo León, México. Esta empresa se encontraba en el proceso de iniciar la construcción de un código de ética durante el año 2009. La empresa contaba con 1,250 empleados, distribuidos en las tres plantas y sus oficinas corporativas.
El proceso de generación del código de ética se documentó en tres fases diferentes:

1. Sensibilización y consulta sobre la pertinencia de un código de ética empresarial

\section{Construcción del código de ética empresarial}

3- Difusión del código de ética empresarial.

Fase 1. Sensibilización y consulta sobre la pertinencia de un código de ética empresarial

Con el fin de contar con una perspectiva amplia de la organización acerca de la relevancia de contar con un código de ética así como las expectativas del mismo, se entrevistaron a 138 participantes que colaboraban en las diferentes áreas de la organización y que ocupaban distintos niveles dentro del área para garantizar la inclusión de participantes de la organización. Lo anterior respalda lo argumentado por Vieira (2012) acerca de que la construcción de un código de ética debe ser el resultado de un proceso colectivo de construcción de normas de comportamiento y reflejar la expresión de la cultura organizacional que todos desean.

La entrevista se estructuró en tres grandes áreas:

1. La relevancia de la ética en la vida organizacional.

2. La valoración de un código de ética.

3. Las expectativas respecto a su diseño e implementación.

Las duraciones de las entrevistas versaron entre 20 y 30 minutos y en el muestreo se incluyeron a 138 participantes que integraban las 8 áreas organizacionales, representando a los 3 niveles organizacionales (directivo/gerencia, profesional de apoyo/jefatura y supervisión/operación). El hecho de incluir a los distintos niveles organizacionales en la consulta, evitó caer en el error señalado por Vieira (2012) de transmitir la idea de que el código es aplicable solo a los trabajadores de cierto nivel. Este error se hace evidente cuando los trabajadores no participan en su definición o se forma un comité de ética que excluye la representación de los trabajadores y solo se incluye a jefes y directivos. Además, se tomó en cuenta que el contacto personal de las entrevistas aplicadas permite una mayor apertura y comprensión de los temores y expectativas en relación con el código de ética (Pou Munt et al. 2014, p. 7)

Tomando como referencia a Ruiz-Olabuénaga $(2007)^{1}$, las respuestas a las preguntas en las entrevistas fueron clasificadas de acuerdo con la incidencia de palabras clave y siguiendo

1. Ruiz-Olabuénaga (2007) refiere que "el análisis de contenido es una metodología que utiliza una serie de procedimientos para efectuar inferencias válidas sobre el autor, el mensaje, la audiencia de un texto” (p. 199). También explica que en tal proceso hay dos momentos clave. El primer momento es la codificación, proceso que implica "la reducción de muchas palabras del texto mediante su clasificación en un número mucho menor de categorías" (p. 199). Y el segundo es el tratamiento de los datos clasificados, que consiste en "el recuento de frecuencias, de palabras, listado de palabras clave en su contexto, concordancias y clasificaciones de palabras en categoría de contenido" (p. 199). 
una clasificación en categoría de contenidos. Por ejemplo la impartición de cursos como estrategia para fomentar la ética (1) fue asociada con otras respuestas similares tales como: talleres de capacitación (1.1), programas de capacitación (1.2), proyectos de capacitación (1.3) cursos de entrenamiento (1.4). De esta forma se siguió el momento de codificación y de clasificación de las respuestas a las entrevistas realizadas. El método de análisis de contenido permitió procesar la información de una manera útil y eficiente. $Y$ con base en ello, entender el mensaje de la organización, a través de las respuestas de sus colaboradores.

Ahora bien, la entrevista aplicada se guió por los aportes de Argandoña (2003), Rodríguez y Díaz (2004), Solomón (1999), Schwartz (2001), Helin y Sandström (2007), Fernández (2001) y Treviño, Hartman y Brown (2000). En las investigaciones de esos autores encontramos los elementos claves y relevantes en relación con los contenidos de los códigos de ética, así como recomendaciones en el proceso de construcción del mismo. Específicamente tales autores señalan la importancia de sensibilizar a la organización acerca de la relevancia y necesidad de la ética en la forma de proceder como empresa. Adicionalmente, los autores argumentan sobre la necesidad de seguir un proceso incluyente en el diseño del código, verificar la aplicabilidad de los criterios éticos establecidos, así como los procesos para asegurar el entendimiento y la apropiación del código de ética a partir de una racionalidad convencida. En consecuencia, la Tabla 2 desvela las preguntas orientadoras con sus respectivas respuestas y frecuencias. Las encuestas representan el primer paso previo para la posterior apropiación del código de ética, cuyo objetivo es encontrar las buenas razones que legitiman la naturaleza de tal esfuerzo.

\section{Fase 2. Construcción del código de ética empresarial}

Una vez realizadas las entrevistas y concentrado los resultados, se llevaron a cabo 5 sesiones para establecer los apartados que debía contener el código de ética y lo que se entendía por cada uno de ellos. Los grupos de discusión estaban integrados por 30 personas, incluyendo los puestos de primer y segundo nivel organizacional como los directivos, gerentes y jefes de las distintas áreas de la organización. Este sentido de colectividad en la construcción de un código de ética fue referido por Rodríguez y Díaz (2004) y señalado por Helin y Sandström (2007) quienes indican que un código de ética corporativo debe estar respaldado por una moral compartida en la organización. Esta estrategia incluyente busca seguir las definiciones establecidas por Cortina et al. (1996) en donde indican que un código de ética logra converger la diversidad de ideas acerca de lo que se entiende como el ethos organizacional.

Adicionalmente, Vieira (2012) advierte que el código de ética no debe de enlistar todas las posibles situaciones a enfrentar sino que debe ser una guía amplia para orientar las decisiones que se toman en la empresa y que involucran a los distintos stakeholders de la organización. También se recomienda que la redacción de los lineamientos sean expresados en positivo de tal forma que invite a su cumplimiento y omitir expresiones autoritarias que provoquen rechazo.

En el caso bajo estudio que aborda la presente investigación, como punto de partida se retomaron los diferentes aportes que fueron compartidos por los colaboradores durante las entrevistas realizadas para hacer una primera propuesta. El proceso de diálogo que se siguió en las sesiones presenciales resultó ser clave para encontrar criterios justos y aplicables a la vida empresarial, gracias a ello se pudo cumplir con una de las recomendaciones propuestas por Vieira (2012) en relación a que un código de ética debe reflejar un proceso colectivo en la definición de normas.

Finalmente la construcción del código concluyó con las siguiente estructura: 1) Introducción al código de ética, 2) Antecedentes, 3) Objetivos, 4) Alcance, 5) Lineamientos éticos generales, 6) Relación con clientes y consumidores, 7) Relación con proveedores, 8) Política de regalos, 9) Relación con la competencia, 10) Relación con los colaboradores, 11) Conflicto de interés, 12) Información confidencial, 13) Protección de activos, 14) Política de seguridad, 15) Política de austeridad, 16) Respeto por las leyes y el medio ambiente, 17 ) Gestión del código de ética.

Las precisiones que se mencionan a continuación son un ejemplo de los enunciados que fueron construidos de forma colectiva e incluyente en las reuniones de directivos, gerentes, y que originalmente fueron esbozadas en la consulta que se realizó a la organización. Estas precisiones buscan clarificar los criterios éticos que deben seguir los colaboradores para responder a temas concretos, como lo son la relación con clientes, proveedores y personal. En la declaración de los criterios éticos se tomó en cuenta la recomendación establecida por Rodríguez y Díaz (2004) acerca de lograr un equilibrio entre criterios éticos muy específicos y criterios éticos muy generalizados en un código de ética. Por ejemplo, en lo que se refiere a la relación con clientes, se estableció "Mantenemos nuestro compromiso por acordar con nuestros clientes solo aquello que podamos cumplir cabalmente y entregar aquello que hemos prometido. Al evitar comprometernos con aquello que no podemos cumplir, mantendremos la confianza y credibilidad en el mercado".

La anterior declaración encuentra su fundamento ético en el apego al deber ser en la relación clienteempresa, respetando ante todo la autonomía del cliente al no sesgar su decisión y que ésta logre ser objetiva y no manipulada (Kant, 1921).

En cuanto a la relación con los proveedores, se definieron los siguientes criterios éticos basados en la universalización de la norma (Kant, 1921): "La selección de los proveedores se basará en criterios de competencia 


\section{Tabla 2. Preguntas de la entrevista sobre el código de ética, respuestas y frecuencias}

¿Qué se hace en la empresa para promover la ética en la cultura de la empresa?

a) Se imparten cursos de capacitación (102)

b) Está incluida en su cultura organizacional, reglamentos y procedimientos de trabajo (37)

c) Se promueve el involucramiento, la participación, la libertad de expresión, la comunicación, el sentido de pertenencia y la congruencia (33)

¿Qué debe hacer la empresa para fomentar la ética en la organización?

a)Continuar con la capacitación en ética y que sea dinámica, comprensible y actualizada (83)

b)Elaboración y seguimiento del código de ética y formar comités de ética (36)

c)Evaluación basada en valores y reconocimiento (o castigo) por su cumplimiento (30)

¿Cuáles son las situaciones de su área en dónde la ética es criterio de decisión y acción?

a)En la administración del personal así como sus responsabilidades y obligaciones (69)

b)En la interacción con clientes y proveedores (64)

¿Cuáles son los principales dilemas éticos que se presentan en su área de trabajo?

a) ¿Hasta dónde intervenir y cómo actuar ante las irregularidades en la relación con los clientes y proveedores? (35)

b)¿Qué posición asumir cuando un compañero no cumple con sus responsabilidades y carece de compromiso? (19)

c) ¿Cómo saber si se es justo y equitativo con el personal y tener claridad en su aplicación? (19)

¿Cuáles son las consecuencias a corto y largo plazo de tomar en cuenta la ética en la empresa?

a) Genera confianza y la aumenta (45)

b)La permanencia del negocio mediante la consolidación y el aseguramiento del éxito, que favorezca una organización sana y fuerte y en crecimiento (33)

¿Qué pasaría si no se respondiera con ética en la organización?

a) Generaría desconfianza interna y externa (53)

b)No permite el crecimiento y se compromete la permanencia del negocio (quiebra y fracaso) (45)

¿Qué temas deberían incorporarse en un código de ética?

a)Conflicto de intereses (51)

b) Relación con clientes y proveedores (48)

c) Valores de la organización y su definición (47)

¿Por qué es importante un código de ética para la empresa?

a) Establece los comportamientos o normas de conducta esperados por la organización (58)

b)Establece lineamientos para clarificar conflictos, dilemas éticos y áreas o zonas grises (36)

c)Formaliza el fomento a los valores y la cultura organizacional (34)

¿Qué beneficios le aportaría hacia el interior y exterior de la empresa un código de ética?

a)Genera, mantiene y fortalece la credibilidad y confianza (58)

b)Guía, fomenta y refuerza el comportamiento y la toma de decisiones basado en los valores (57)

c)Mantenerse como organización consolidada (40)

¿Qué requisitos deberían considerarse para que un código de ética sea eficaz en una empresa?

a) Que se difunda y cumpla en todas las áreas, niveles organizacionales clientes y proveedores (77)

b)Que se le dé seguimiento, sea medible, que se revise y actualice (39)

c) Que sea claro y conciso (38)

¿Qué aspectos se deben tomar en cuenta en su elaboración y diseño?

a)Que la redacción sea clara y concisa y fácil de entender (48)

b)Que sea acorde con la situación, necesidades y perfil de la empresa (38)

c) La participación del personal (opiniones, ideas) (31)

¿Qué consideraciones se deben atender para su debida implementación y seguimiento?

a)Una adecuada promoción y difusión en todas las áreas, niveles, clientes y proveedores (62)

b)El adecuado seguimiento, involucramiento y compromiso para cumplimiento del código (50)

c)La evaluación de su aplicación y la actualización (revisión) (24)

Fuente: Arredondo, De la Garza y Villa (2014).

justa, sin buscar obtener ni otorgar un beneficio en la asignación de contratos o en la adquisición de bienes y servicios. Para ello tomaremos como factores de la decisión la calidad del producto, el precio, así como la calidad en el servicio y el cumplimiento de entrega. Estos factores deberán ser ponderados buscando optimizar de forma objetiva el beneficio de la organización".

En relación con el trato con el personal se especificaron criterios éticos centrados en el respeto a la dignidad humana que encuentran sustento en la perspectiva kantiana (Kant, 1921), que considera a la persona como fin en sí misma y no sólo como medio: "Quienes laboramos en la empresa nos comprometemos a respetar a las personas en el trato diario, por lo que se prohíbe cualquier tipo de acoso, prácticas de discriminación, favoritismo o cualquier tipo de comportamiento que ofenda la dignidad de las mismas o les provoque daño moral a personas internas o externas vinculadas con la organización". 


\section{Fase 3. Difusión del código de ética empresarial}

En relación con la difusión del código de ética empresarial, Argandoña (2003) aborda la relevancia de comunicar, institucionalizar y comprometer los principios éticos declarados en los códigos de ética. Además, incluye la importancia de integrarlo a los proyectos de capacitación como parte de la difusión de este tipo de estrategias. En relación con la difusión y actualización del código, se atendieron los señalamientos presentadas por Arredondo et al. (2014), en donde se sugiere una revisión y actualización contínua de tales códigos.

Para Pou Munt et al.(2014, p. 12) se debe formar un comité representativo a cargo de la implementación y posterior evolución del código de ética. Tanto la difusión como la retroalimentación debe involucrar activamente al personal así como el aseguramiento de la comprensión del código mediante cursos de capacitación.

En el caso bajo estudio se propuso que para comunicar el código de ética se tendría que entregar un ejemplar de forma personalizada a cada colaborador para proceder a su firma y con ello ratificar el compromiso por su cumplimiento en una ceremonia presidida por el director general en la que se llevaría a cabo la entrega formal del código. Además, cada directivo se comprometió a replicar la sesión de entrega de códigos en sus plantas o áreas funcionales, con la presencia del director general. Las cartas compromiso se planearon recolectar por parte de la dirección de recursos humanos. Y finalmente, se propuso entregar un ejemplar a cada proveedor para ser firmado, así como enviar un ejemplar del código a los clientes con una carta personalizada.

Para asegurar la vinculación del código de ética a la vida organizacional, se diseñaron mini-casos en boletines en donde se veía la aplicación del código por áreas organizacionales, además de hacer mención al código en el reglamento de trabajo.

Finalmente, para difundir el código se diseñaron protectores de pantalla en donde se promovía el contenido del código, también se procedió a publicar posters del código en lo referente a proveedores en áreas estratégicas dentro de la empresa, elaborar un documento resumido del código y diseñar un poster para difundir el código en plantas y oficinas, e invitar a conocerlo, y subir el código a la página web de la empresa.

\section{Resultados y discusión}

Tal como se establece en la teoría de la ética organizacional en la que se hace referencia a los códigos de ética, en este caso de estudio, es visible que una de las formas para integrar la ética en la organización son los procesos de formación del personal. La capacitación se propone por los propios colaboradores como un proceso clave, que debe llevarse a cabo antes de la construcción de un código para sensibilizar a la organización.
Adicionalmente, la teoría sobre la ética empresarial declara que la ética debe cubrir tanto la parte interna del quehacer de la organización como la parte externa. En el estudio se menciona la relevancia que tiene la ética en la gestión del personal, así como la relación con clientes y proveedores, confirmando este doble enfoque. Por otra parte, respecto al código de ética, los participantes en el estudio mencionaron que todo código debe cubrir principalmente, pero no exclusivamente, lo que se refiere a los clientes, proveedores y gestión del personal. Hay una necesidad por aclarar cómo se deben de establecer estas relaciones en la vida organizacional.

Todo este proceso de consulta parte de la base de respetar la autonomía de los colaboradores en donde se expresa racionalmente su voluntad humana, tomando como base la perspectiva ética kantiana en el marco teórico.

También desde la ética empresarial se refiere que todo código de ética debe ser útil para la toma de decisiones, en particular cuando se enfrentan cuestionamientos dilemáticos en donde no es claro qué es lo que se debe hacer en una situación particular. En relación con ello, la empresa bajo estudio, declaró que aclarar conflictos con clientes y proveedores es el tipo de dilemas que deben de guiar los códigos de ética, además de orientar la relación entre el personal y el trato equitativo y justo entre quienes colaboran en la organización. En la aclaración de tales conflictos de interés, los acuerdos tomados por la organización encontraron su fundamento en la universalización de las normas desde una ética kantiana, tal como se especificó en el marco teórico.

Es interesante que los colaboradores que participaron en el estudio mencionen a la confianza y la permanencia del negocio como dos valores derivados de guiarse en una ética organizacional. La lectura de la organización respecto a este tema, es que la ética da confianza y sin confianza se compromete la viabilidad de una empresa. Esta perspectiva de ver la ética organizacional, vinculada estrechamente con el capital de confianza, se ve como una inversión, no solo en el quehacer presente de la vida organizacional, sino en el futuro, al garantizar la permanencia del negocio. Llama la atención que el consejo directivo haya considerado la dimensión de la ética organizacional como un pilar para asegurar la viabilidad de la industria en el largo plazo.

En relación con los elementos a cuidar en el diseño y la implementación de un código de ética, se comprueba que estos deben ser consensuados para responder a las necesidades de la organización. Los elementos que contenga el código deben ser clarificadores, es decir, deben ser útiles para la operación diaria, deben difundirse, aplicarse y actualizarse. Finalmente, como remarcan distintos autores de la ética organizacional, en el caso de estudio se detecta la relevancia de predicar con el ejemplo, de ser congruentes y no engañar, tal con se infiere en la postura kantiana en relación con la no instrumentalización de la 
persona. Así como apelar a la racionalidad convencida para la debida apropiación de las normas éticas que contempla el código.

A partir de la experiencia de diseño de un código de ética se propone el modelo (Figura 1) para la creación de un código de ética empresarial que pueda ser útil para otras empresas que tengan la necesidad de fundamentar este proceso desde una base filosófica ética como es la propuesta kantiana.

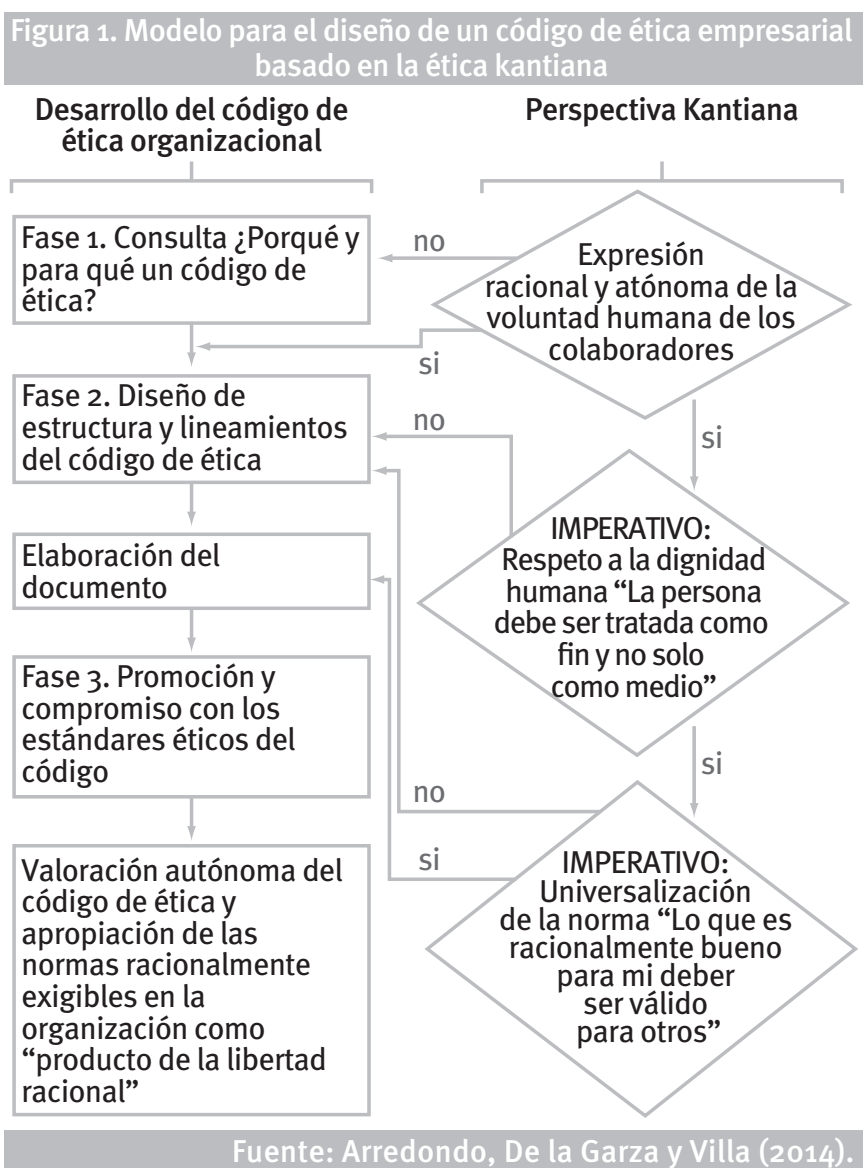

\section{Conclusión}

La intención de definir un código de ética para una empresa debe surgir, no de una imposición de la élite directiva y sí de la convicción colectiva de tener claro por qué es importante la ética y cuáles deben ser los lineamientos éticos a los que debe ajustarse la organización. En relación con la implementación de los códigos, hay diferencias si estos se imponen o se asumen. Quienes colaboran en una empresa, no solo se dirigen por leyes o normas estrictas, sino por normas entendidas y asumidas como parte de sus convicciones, normas que poseen una relevancia para la persona como para la organización, y que gracias a ellas se construyen estándares éticos que dan sustento al deber ser de la organización.

Se concluye además que el diseño de un código de ética debe fundamentar sus bases en la ética kantiana, estableciendo con claridad y precisión el deber ser de la organización. En su construcción es mandatorio incluir las voces de quienes laboran en la organización y en su diseño se debe cuidar la aplicabilidad del mismo. De esta forma, el código de ética logra responder a los problemas que todo colaborador se enfrenta en la vida organizacional, así como asegurar su legitimidad como documento rector ante la propia empresa y el entorno.

Si bien en el contexto de la ética empresarial se han sugerido diversas metodologías para la construcción de códigos de ética, la novedad que aporta esta investigación es el sustentar el proceso del diseño de su contenido en dos principios rectores que vienen siendo los imperativos kantianos. Los criterios parten de un proceso racional guiado por el respeto a la dignidad humana en la forma de relacionarse con otros; así como en la universalización de las normas, para asentar criterios justos, es decir, que no solo convengan parcialmente a la organización. Ambos principios sustentan y fundamentan los argumentos que derivan de un código de ética, apelando a una racionalidad convencida, requisito básico en la elaboración y adecuada implementación de todo código de ética. El proceso seguido garantiza además que las normas emergidas no resulten ser arbitrarias o simplemente replicadas de otro código ya existente, sino proveniente de la autonomía de los colaboradores.

Como parte de la capitalización de este ensayo, se propone un modelo que puede ser referenciado por investigadores que se interesen en estudiar la forma en que las empresas construyen sus propios códigos de ética empresarial, bajo este sustento filosófico. Una de las principales limitantes es que no se cuenta con un diagnóstico previo para medir posibles cambios en la ética organizacional antes y después de la aplicación del código de ética.

\section{Referencias}

Argandoña, A. (2003). Private-to-private corruption. Journal of Business Ethics, 47 (3), 253-267.

Aristóteles (2006). Ética a Nicómaco. (V. Gutiérrez, Trad., 2003). Madrid, España: Mestas.

Arredondo, F., De la Garza, J., \& Vázquez, J. C. (2014). Transparencia en las organizaciones, una aproximación desde la perspectiva de los colaboradores. Estudios gerenciales, 30 133, 408-418.

Comte-Sponville, A. (2009). El capitalismo ¿es moral? Barcelona, España: Paidós.

Cortina, A., Conill, J., Domingo, A., \& García, D. (1996) Claves para una cultura empresarial: ética de la empresa. Madrid, España: Editorial Trotta.

Cortina, A. (2003). Razón pública y éticas aplicadas los caminos de la razón práctica en una sociedad pluralista. Madrid, España: Editorial Tecnos.

Fernández, J. (2001). Elementos que consolidan el concepto de profesión. Revista electrónica de investigación educativa, 3 (2), 23-39.

Gillén, M. (2006). Ética de las organizaciones: construyendo confianza. Madrid, España: Prentice Hall. 
Gilligan, C. (1982). In a different voice: Psychological theory and women's development. Cambridge, Estados Unidos: Harvard University Press.

Gopinath, C. (2008). Recognizing and justifying private corruption. Journal of Business Ethics, 82 (3), 747-754.

Habermas, J. (2008). La inclusión del otro. Barcelona, España: Paidós.

Hamilton, S., \& Francis, I. (2003). The Enron collapse. International Institute for Management Development, 13 (2), 135-144.

Hartman, L., Desjardins, J., \& Espinoza, F. (2011). Ética en los negocios. Decisiones éticas para la responsabilidad social $e$ integridad personal ( ${ }^{\mathrm{da}} \mathrm{ed}$.). México, D.F., México: McGrawHill.

Helin, S. \& Sandström, J. (2007). An inquiry into the study of corporate codes of ethics. Journal of Business Ethics, 75(3), 253-271.

Ibarra, R. (2002). Código de ética, cómo implementarlo en la empresa. México, D. F., México: Ed. Trillas.

Jonas, H. (1993). El principio de responsabilidad, ensayo de una ética para la civilización tecnológica. Barcelona, España: Editorial Herder

Kant, I. (1921). Fundamentación de la metafísica de las costumbres. Madrid, España: Calpe.

Longinos, J., Arcas, N., Martínez, I., \& Olmedo, I. (2012). Transparencia, Gobierno Corporativo, y Participación: Claves para la implantación de un código de conducta en empresas de economía social. Revista de Estudios Cooperativos, (108), 86-112.

Lozano, F. (2004). Códigos éticos para el mundo empresarial. Madrid, España: Trotta.

Melé, D. (2014). Diez recomendaciones para el rearme moral de la banca. Ética empresarial. Recuperado 25/10/2014 de http://blog.iese.edu/eticaempresarial/author/dmele/

Mendes, E., \& Clark, J. (1996). The five generations of corporate codes of conduct and their impact on corporate social responsibility. Recuperado 10/02/2014 de http://www. toronto.ca/inquiry/inquiry_site/cd/gg/add_pdf/77/Conflict_of_Interest/Electronic_Documents/Research_orgs/ Five_Generations.PDF
Mill, J. S. (2002). El utilitarismo. Esperanza Guisán. Madrid, España: Alianza

Porter, M., \& Kramer, M. (2006). Strategy \& society: the link between competitive advantage and corporate social responsibility. Harvard Business Review. Mass, 84(12), 78-92.

Pou Munt, G., Fabbri, M., Chamón, J., Villavicencio, R., y Schmidt, E. (2014). Nuestro modo de proceder: Una nueva forma de concebir y desarrollar códigos de ética profesional en América Latina. Recuperado 25/10/2014 de http://www. fidamerica.org/admin/docdescargas/centrodoc/centrodoc_450.pdf

Rawls, J. (2006). Teoría de la justicia. México, D.F., México: Fondo de Cultura Económica.

Rodríguez, M. P., y Díaz, A. F. (2004). Códigos Éticos: construcción colectiva del carácter organizacional. El caso de la Universidad Nacional de Colombia sede Manizales. Innovar, 24 (28), 39-58.

Ruiz-Olabuénaga, J. I. (2007). Metodología de la investigación cualitativa ( $4^{\text {ta }}$ ed.). Bilbao, España: Universidad de Deusto.

Schwarz, M. S. (2001). The nature of the relationship between Corporate Code of ethics and behaviour. Journal of Business Ethics, 32 (3), 247-262

Schwarz, M. S. (2004). Effective corporate code of ethics: Perceptions of code users. Journal of Business Ethics, 55 (4), 323-343.

Treviño, L., Hartman, L., \& Brown, M. (2000). Moral person and moral manager: How executives develop a reputation for ethical leadership. California Management Review, 42 (4), 128-142.

Velázquez, M. (2010). Business ethics. New Jersey, USA: Pearson Education.

Vieira, C. (2012). Código de Ética: Mucho más que buenas intenciones. Ética Pública y Lucha contra la Corrupción, Recuperado 25/10/2014 de http://www.gestionpublica. org.pe/plantilla/rxv5t4/1029474941/enl4ce/2012/jun/ revges_1561.pdf

Villa, L. (2011). Capitalismo y empresa: reflexiones desde la ética y la RSE. Bogotá, Colombia: Pontificia Universidad Javeriana

Weis, J. (2011). Ética en los negocios. México, D.F., México: Thompson.

Cuadernos de Administración / Facultad de Ciencias de la Administración / Universidad del Valle

Periodicidad: semestral / ISSN impreso $N^{\circ}$ 0120-4645 - ISSN electrónico N ${ }^{2256-5078 ~ / ~ N o m b r e ~ a b r e v i a d o: ~ c u a d . a d m . ~}$

Edición Vol. $30 \mathrm{~N}^{\circ} 52$ (julio - diciembre de 2014)

Propuesta para el diseño de un código de ética empresarial basado en la ética kantiana / Florina Guadalupe Arredondo Trapero, Lida Esperanza Villa Castaño y Jorge De la Garza García

\section{(c) (1) (8)}

Revista Cuadernos de Administración por Universidad del Valle se encuentra bajo una Licencia Creative Commons Atribución-NoComercial-

Compartirlgual 2.5 Colombia.

Basada en una obra en http://cuadernosdeadministracion.univalle.edu.co/ 